\title{
Comfort in observing stereoscopic images reduced by vibration stimuli
}

\author{
Hiroshi Watanabe ${ }^{1^{*}}$, Hiroyasu Ujike ${ }^{2}$ \\ ${ }^{1}$ Health Research Institute, National Institute of Advanced Industrial Science and Technology (AIST), Osaka, Japan; \\ *Corresponding Author: h.watanabe@aist.go.jp \\ ${ }^{2}$ Human Technology Research Institute, National Institute of Advanced Industrial Science and Technology (AIST), Ibaraki, Japan
}

Received 28 August 2012; revised 3 October 2012; accepted 19 October 2012

\begin{abstract}
Numerous studies have been conducted to illuminate the effect of image factors to reduce unexpected influence of stereoscopic images on healthy visual experience. In this paper, we introduce changes in the psychological and physiological indexes of observers of a stereoscopic image disturbed by vibration stimuli. Forty-four healthy university students participated in the experiment. A programmable vibration table generated two types of vibrations (5 Hz and $20 \mathrm{~Hz}$ ) and provided intermittent vibration stimuli to a stereoscopic projector installed on a vibration table. Our results showed that the frequency of vibration stimuli has a strong impact in evaluating the local comfort of subjects. Our results also showed that the indexes of visual fatigue increased after observation independent of the frequency. The activity status of the autonomic nervous system as a physiological index significantly increased after observing 3D images with vibration stimuli although the vibrational frequency did not have a significant effect on the activity status.
\end{abstract}

Keywords: 3D Movie; Vibration Stimuli; Visual Fatigue; Heart Rate Variability; Subjective Comfort Evaluation

\section{INTRODUCTION}

The development of display technologies has made the observation of stereoscopic images more accessible. In recent years, we have been able to enjoy stereoscopic images not only at home but also outdoors using portable video equipment (mobile phones, smartphones, game equipment, etc.). Here, the issue in observing stereoscopic images in such a dynamic environment is unexpected vibration given to the images. Stereoscopic imag- ing devices input the different images to both eyes according to the horizontal disparity. The visual information processing system in the brain fuses those images to enable the observer to perceive a stereoscopic effect. Thus it is preferable that both the projection system and the observer are in stationary condition. Even under this stationary condition, it is known that comfortable observation of stereoscopic images is inhibited by various factors including unexpected rotation, geometric distortion, vertical shift, horizontal shift, color shift, difference in brightness and so on between left and right eyes [1-11]. It is considered necessary to examine the kind of discomfort that occurs under the condition where both the display and the observer move. Some previous studies examined the comfort or discomfort that vibration stimuli themselves give to the human body [12], but there is no previous study on the effect of body vibration on observation of the stereoscopic images.

Only a few studies discussed that the biomedical effect of common 3D movies (dynamic) on observers; Speranza et al. [6] studied accommodative asthenopia using with simply oscillated objects in depth direction. Yano et al. [8] presented a common 3D movie to observers and estimated the effect of disparity changing included in the movie on the function of accommodation. In our study, on the other hand, we presented vibration stimuli irrelevant to contents of the movie for disturbing fusion right and left images to obtain 3D perception. This procedure assumed the daily life situation in which unexpected vibration stimuli possibly occur with observing stereoscopic image such as in mobile display. We also measured both psychological and physiological indexes continuously under such a successive observation, and this is another appeal point of this paper.

In this experiment we presented the observation disturbing factor, which does not exist during typical stereoscopic image observation, by placing a stereoscopic image projector on a vibration table. Two types of vibration stimuli, relatively high $(20 \mathrm{~Hz})$ and low $(5 \mathrm{~Hz})$ frequencies, were provided to the stereoscopic images. 
All subjects observed the images provided with two types of vibration stimuli in random order, taking approximately a one-hour break between the two observations. Two types of psychological reactions and one physiological reaction were measured while the subjects observed an approximately twenty-minute stereoscopic movie. The psychological reaction was measured using a questionnaire (SSQ, [13]) on subjective symptoms concerning motion sickness before and after observation. The other psychological measurement was conducted on subjective symptoms concerning the comfort level, which was asked every two minutes during the observation of the movie. Physiological reaction was measured based on the activity status of the autonomic nervous system derived from electrocardiograms, which were continuously measured during observation.

\section{METHODS}

\subsection{Subjects}

Forty-four male and female university students (average age: $22.36 \pm 1.78$ years old) participated in the experiment. All subjects received payment for their participation. The subjects did not know the purpose of the experiment. Written consent was obtained from each subject for participation in the experiment before starting the study. The details of experiment were approved by the Human Engineering Ethics Committee.

\subsection{Apparatus}

The experiment used commercially available Blu-ray disc software for a marine documentary movie (Deep Sea, Warner Home Video). The movie was reproduced using a Blu-ray disc player (BDP-S470, SONY) and projected shown onto a hundred-inch screen using a 3D projector (VPL-VW90ES, SONY) based on a liquid crystal shutter glasses method. Sound was provided to subjects through headphones from the Blu-ray disc player. A 3D projector was set on a vibration table (BF-50UT, IDEX, Figure 1) to provide vibration stimuli. The apparatus enables vibration stimuli to be reproduced in the directions of $\mathrm{x}, \mathrm{y}$ and $\mathrm{z}$ axes at right angle by rotating a cylinder inside. Programmable setting by seconds is possible for the frequency, start time and stop time of vibration stimuli. Figure $\mathbf{2}$ shows the frequencies of vibration derived from frequency analysis of acceleration information during the experiment. During observation electrocardiograms were derived using Ag-Cl electrodes attached to the chest and abdomen of the subjects and recorded using BIOPAC Systems (Monte System). The sampling frequency was $1 \mathrm{KHz}$. Subjective evaluations on comfort, which were asked every 2 minutes during observation, were also recorded using a digital switch of BIOPAC Systems.

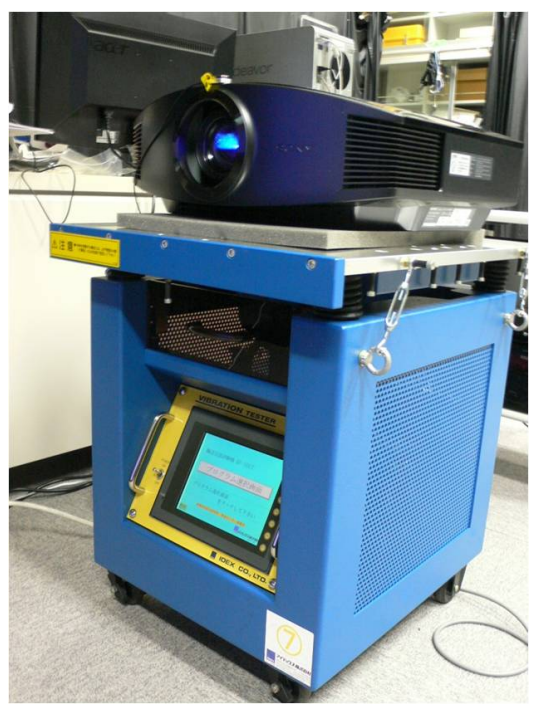

Figure 1. A vibration table and a stereoscopic image projector.
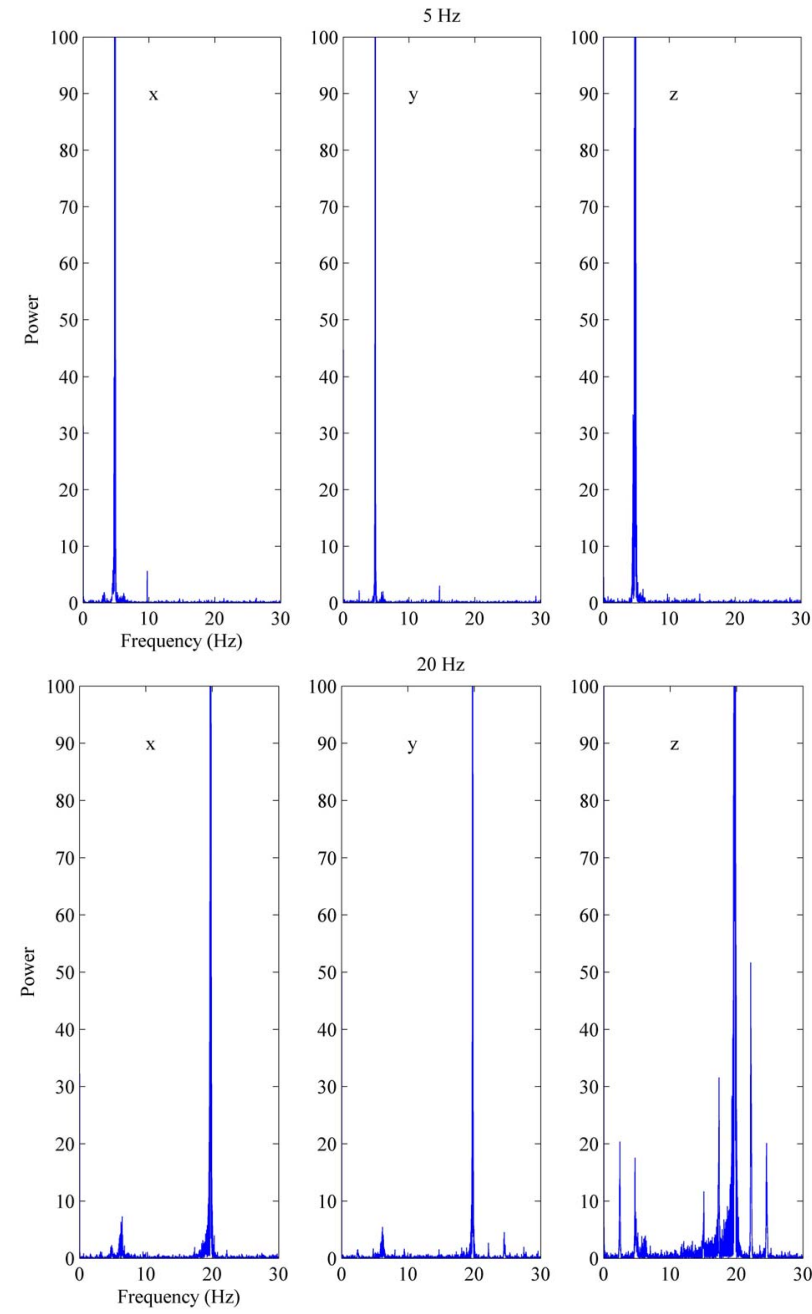

Figure 2. The spectrogram of acceleration in the directions of three axes obtained by FFT of thevibration stimuli adopted in this experiment. 


\subsection{Procedure}

The experiment was conducted in a darkened room. After attaching the electrodes for the electrocardiogram, subjects sat at a distance of $3.74 \mathrm{~m}$ from the screen. This distance complies with a guideline for maintaining comfort when observing stereoscopic movies [14] and it corresponds to three times the height of the screen. All subjects observed the same stereoscopic movies provided with vibration stimuli of two different frequencies $(5 \mathrm{~Hz}$ and $20 \mathrm{~Hz}$ ) in random order. Single observation time was approximately twenty minutes and subjects rested for approximately one hour between the first and second observations. Vibration stimuli were provided five times every two minutes and thirty seconds from five minutes after the beginning of the movie. The vibration durations were five seconds in $5 \mathrm{~Hz}$ and fifteen seconds in $20 \mathrm{~Hz}$. These stimuli were confirmed to provide qualitatively different impressions to subjects in the preliminary study where the first author and three naive subjects participated. $5 \mathrm{~Hz}$ vibration stimulus slowly and greatly vibrated the whole screen. $20 \mathrm{~Hz}$ vibration stimulus, on the other hand, caused fine vibration, which blurred the whole screen. In both cases, the stereoscopic effect was not lost.

The subjects were asked to complete a simulator sickness questionnaire (SSQ) before and after viewing the twenty-minute movie as one of the psychological evaluations on a stereoscopic movie. The SSQ questionnaire consisted of 18 questions and four choices of answers (none, slight, moderate, severe) on movie sickness. The internal status of the observer can be outlined in three categories (nausea, eye fatigue and disorientation) and total scores by placing a specified weight on the raw score.

A direct evaluation of comfort while observing the stereoscopic movie on a five-level scale (very uncomfortable, rather uncomfortable, neither, rather comfortable, very comfortable) was conducted as the other psychological evaluation. The subjects indicated their subjective evaluation in response to a beep sound every 2 minutes and 30 seconds from 10 seconds after the start of movie using a switchbox with five channels placed on their knees.

Figure 3 shows the time schedule of these vibration stimuli and the subjective rating. The broken lines indicate the timing of the subjective rating and the width of the rectangular solid indicates the duration of the vibration stimuli.

\subsection{Statistics}

Differences in mean psychological (SSQ and subjecttive rating) and physiological (autonomic nervous system) index according to vibration frequency basically were examined by analysis of variance (ANOVA). A statisticcal analysis was made by "Matlab Statistics ToolBox (Mathworks, Massachusetts, USA)”. p values were decided depending on $\mathrm{F}$ values and degree of freedom (represented as " $F$ (degree of freedom) $=\mathrm{F}$ value") and the $\mathrm{p}$ values less than 0.05 were considered significant otherwise was non significant (represented as “n.s.”).
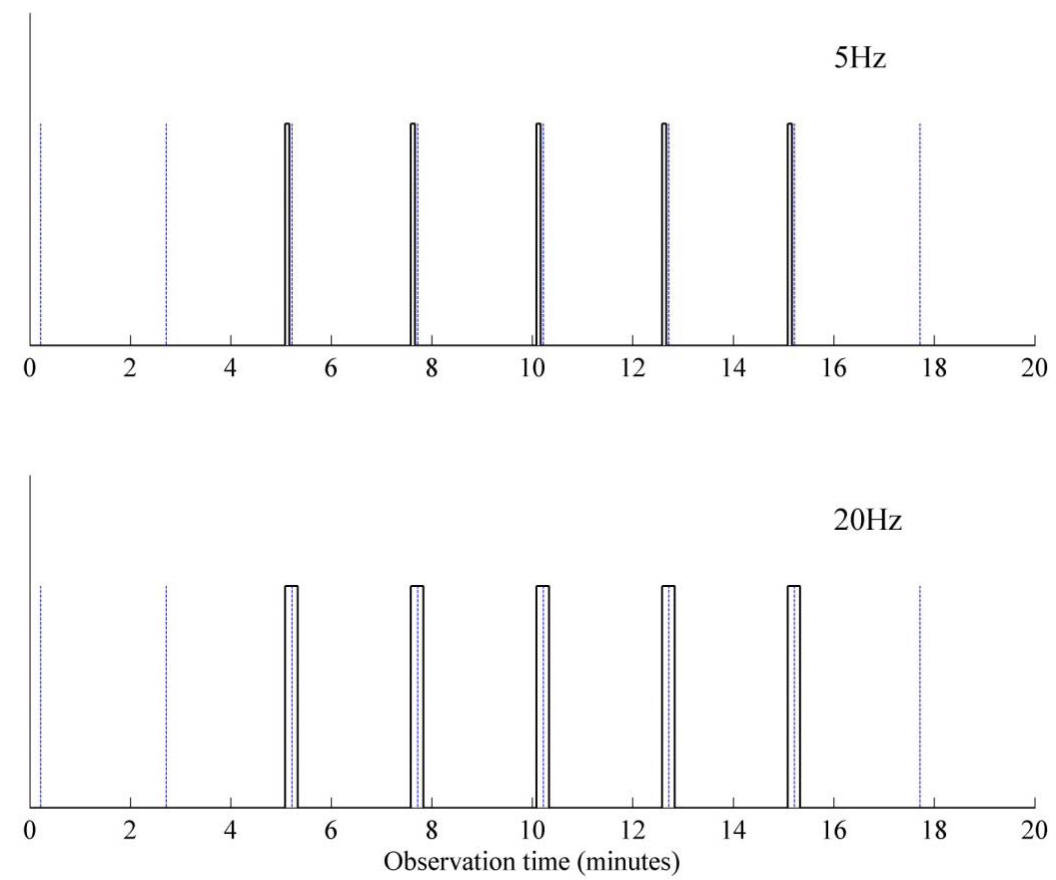

Figure 3. Schedule for presentation of the vibration stimuli and the comfort evaluation (see the text). 


\section{RESULTS AND DISCUSSION}

\subsection{Subjective Ratings}

The scores in SSQ entered by subjects were summarized in four categories (nausea, eye fatigue, disorientation and total score), and we calculated difference between before and after watching the movie in twenty minutes (Figure 4). A two-factor repeated-measure ANOVA was conducted in order to validate the differences of SSQ scores between two frequency factors and among four category factors. The results of ANOVA showed that the frequency factors did not have significant main effect $(F(1,45)=0.46$, n.s. $)$, but there were significant differences in the category of SSQ $(\mathrm{F}(3,14)=10.79, \mathrm{p}<0.01)$. The results of multiple comparisons among categories based on Ryan's method showed that the score for nausea, regardless of frequency factors, was significantly lower than the other three scores (eye fatigue, disorientation, total score: $t=5.52$, 3.80 , and 2.54 respectively, $\mathrm{p}<0.05$ ), and the score for eye fatigue was significantly higher than the total score $(\mathrm{t}=2.98, \mathrm{p}<0.05)$.

These mean values of SSQ changing between before and after observation seemed to be relatively higher than observation normal (without vibration stimuli) 3D cinema. For example, a previous study [15] examined observers' subjective comfort watching a stereoscopic $3 \mathrm{D}$ version of the film Avatar for 165 minutes. Their results showed that mean SSQ changing were approximately $1.9,6.9,8.1$ in nausea, oculomotor, and disorientation, respectively. Thus our results suggested additional irrelevant vibration stimuli on $3 \mathrm{D}$ movie caused the increase of symptom.

Figure 5, on the other hand, shows comfort reported by subjects every two minutes, which is represented as a function of observation time under two frequency conditions. A two-factor repeated-measure ANOVA was used to validate the differences of subjective rating between frequency factors and among observation times. Results of ANOVA showed significant main effects of both frequency factors and observation time $(\mathrm{F}(1,86)=$ 16.14, $\mathrm{p}<0.01, \mathrm{~F}(8,69)=27.91, \mathrm{p}<0.01$, respectively $)$. As interaction of these factors was also significant $(\mathrm{F}(8,69)=5.65, \mathrm{p}<0.01)$, analysis of simple main effects was conducted. The results revealed the comfort level was significantly lowered in the interval between six and fourteen minutes after starting observation under vibration stimuli condition of $5 \mathrm{~Hz}$.

These results suggested the following two properties concerning the subjective states while observing 3D movies provided with vibration stimuli: 1) subjective state relating to eye fatigue (and disorientation) was constantly perceived regardless of vibration frequency, and 2) (local) comfort rating within sort term was clearly affected by vibration frequency. It was suggested that this property was salient when the amplitude was large even though the frequency was relatively low.

\subsection{Physiological Index}

Secondly, we focus on the status of the autonomic nervous system of the subjects while observing the movies. The peak time of each beat was calculated from an electrocardiogram of the subjects while observing the

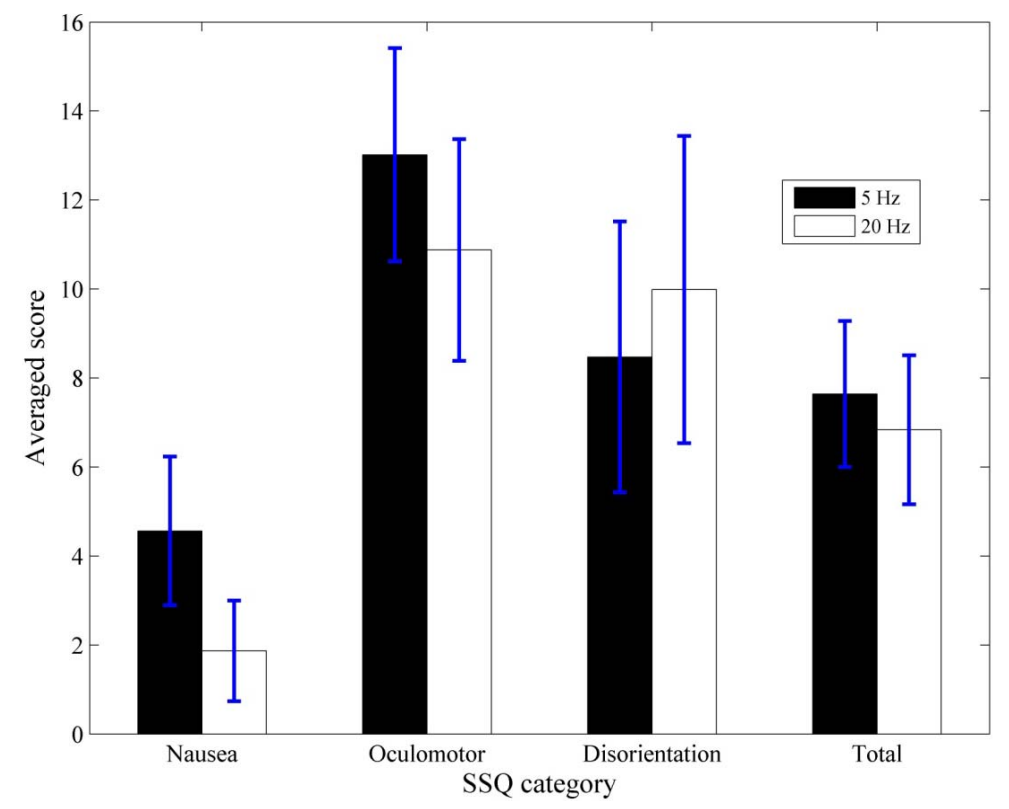

Figure 4. Difference of SSQ scores between before and after observing the movie (average of all subjects). Error bars indicate 1SE. 


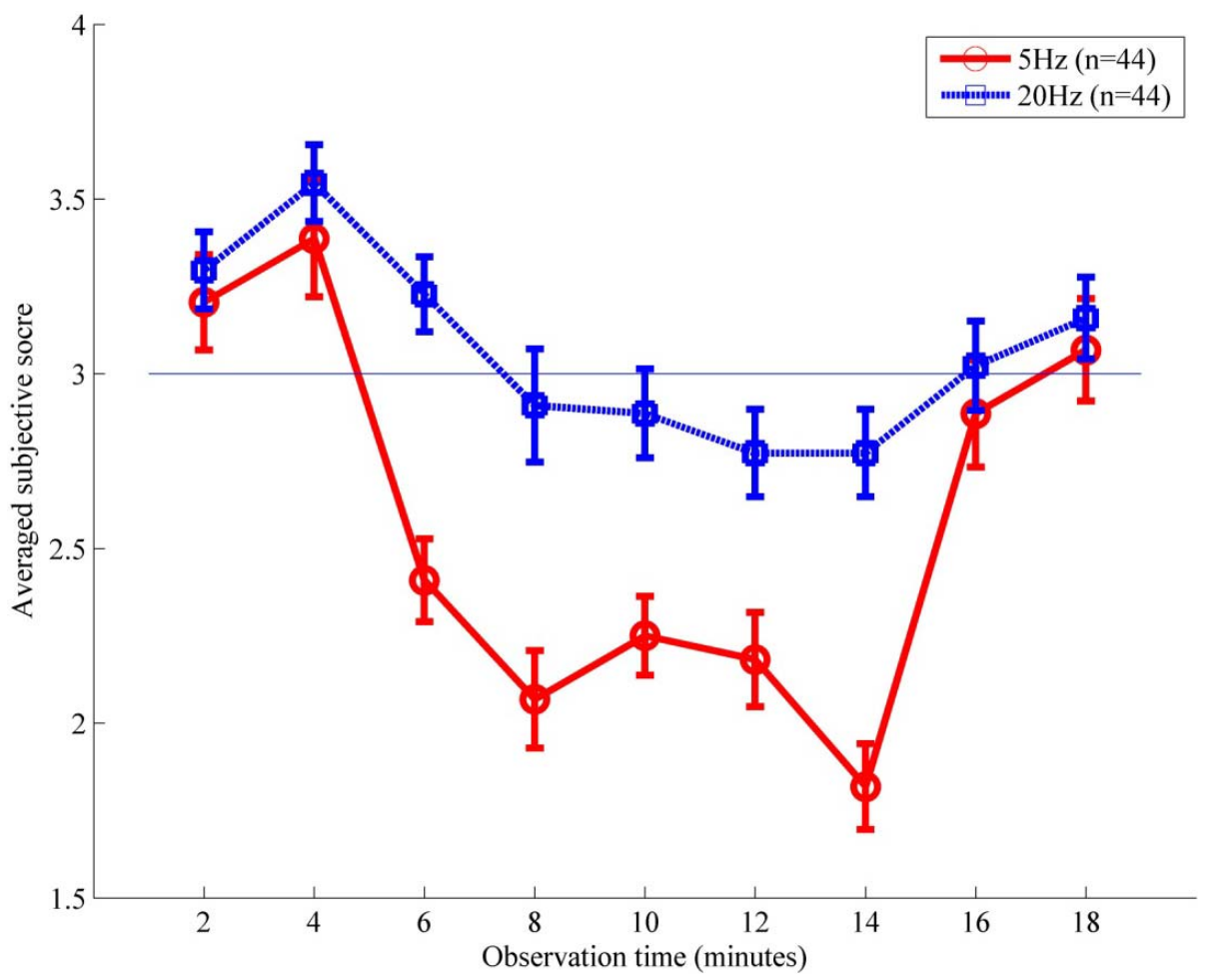

Figure 5. Time series variation of comfort evaluation while observing the movie. Error bars indicate 1SE. Score " 3 " indicates "Neither" in the comfortable scale. The upper area above " 3 " is the comfort area and the lower area below " 3 " is the discomfort area.

movie, and the time interval between adjacent peaks (R-R interval) was calculated. Also, frequency analysis of the R-R interval as a function of time was conducted to calculate the value (LF/HF ratio), which was obtained by dividing the area of the region with frequency of 0.15 $\mathrm{Hz}$ or lower (LF component) by that of the region with frequency of $0.15 \mathrm{~Hz}$ or higher (HF component). The time series variation in the LF/HF ratio was obtained by calculating the LF/HF ratios every one minute from the beginning of observation, using ECG data for three minutes from each calculating time. The LF/HF ratio is considered an index reflecting the activity status of the sympathetic nervous system and the parasympathetic nervous system, which constitute autonomic nervous system, and higher values suggest predominant activity of the sympathetic nervous system.

Next we discuss the interaction with the susceptibility to motion sickness for analysis. We divided subjects into two groups (the average value or higher, less than the average value) using average value of the total scores of SSQ analyzed in Section 3.1. Figures 6 and 7 show the time-series change in the LF/HF ratio in the two groups. We conducted two-factor repeated-measure ANOVAs using vibration frequency and observation time as factors for each group. The results showed the time-series main effects in both groups (high SSQ group: $\mathrm{F}(17,51)=3.64$, $\mathrm{p}<0.01$; low SSQ group: $\mathrm{F}(17,90)=5.84, \mathrm{p}<0.01)$.
Multiple comparison test results suggested that the LF/HF ratios at approximately thirteen to fourteen minutes after the beginning of observation were higher than those at (approximately one to three minutes after) the beginning of observation. The effects of vibration frequency, on the other hand, were not seen in either group (high SSQ group: $\mathrm{F}(1,30)=1.23$, n.s.; low SSQ group: $\mathrm{F}(1,53)=0.42$, n.s. $)$.

The LF/HF ratio itself, however, was consistently relatively high in the frequency of $5 \mathrm{~Hz}$ in high SSQ group but such a difference was not seen in the low SSQ group. Our findings show that in the future we need to clarify the effect of susceptibility to motion sickness and vibration frequency on the autonomic nervous system through the classification by subjects' attribution.

\section{CONCLUSIONS}

We measured the psychological and physiological indexes of observers for twenty minutes during observation of a stereoscopic movie given the vibration stimuli. The subjective data showed that the frequency of vibration stimuli had strong effect on the subject's local comfort evaluation.

However it was indicated that the index of visual fatigue increased after observation of the movie regardless of frequency. Also physiological data showed that the 


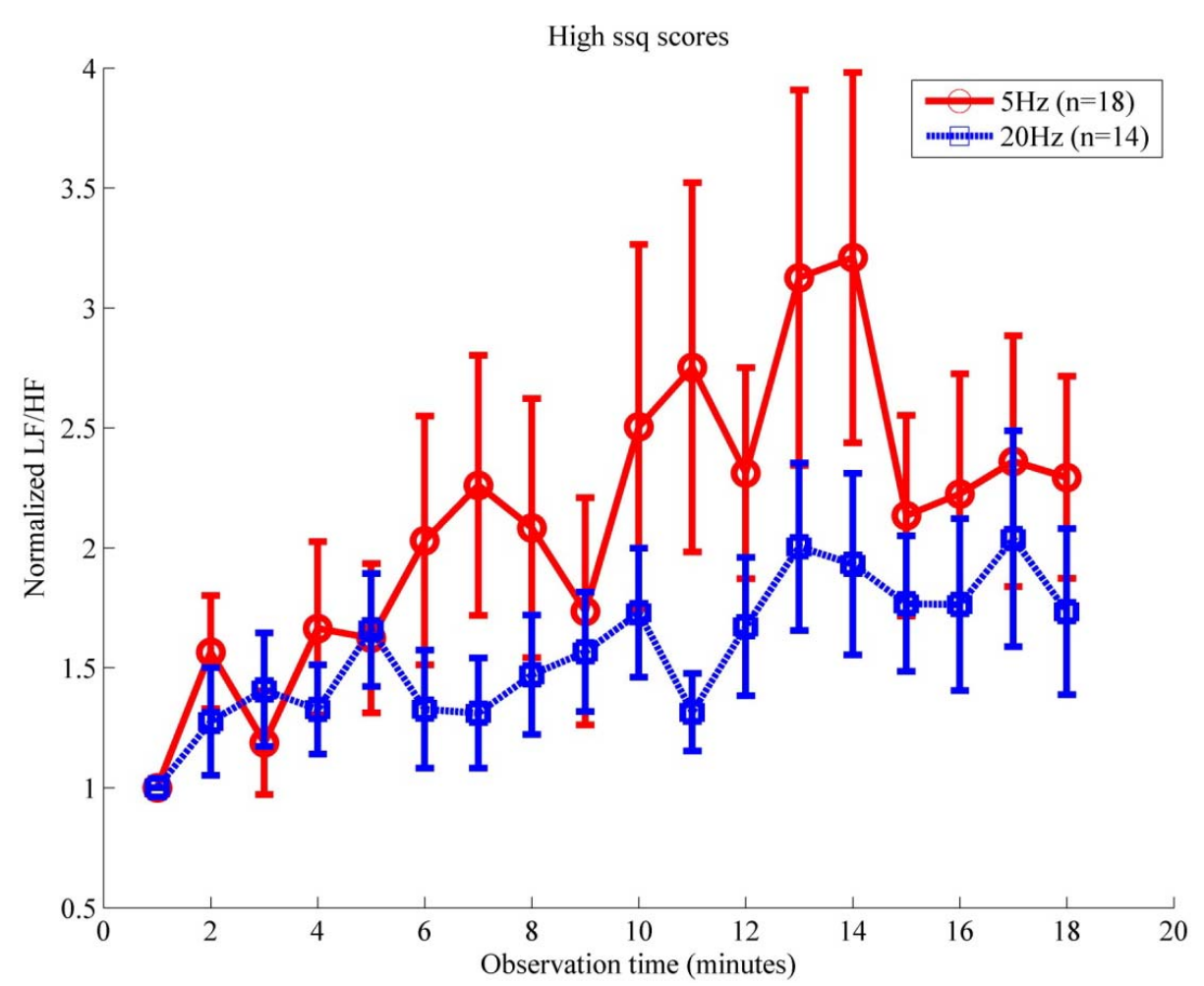

Figure 6. Change in the activity status of autonomic nervous system (LF/HF ratio) while observing the movie. Error bars indicate 1SE. Data of the subjects whose total scores of SSQ were average or higher.

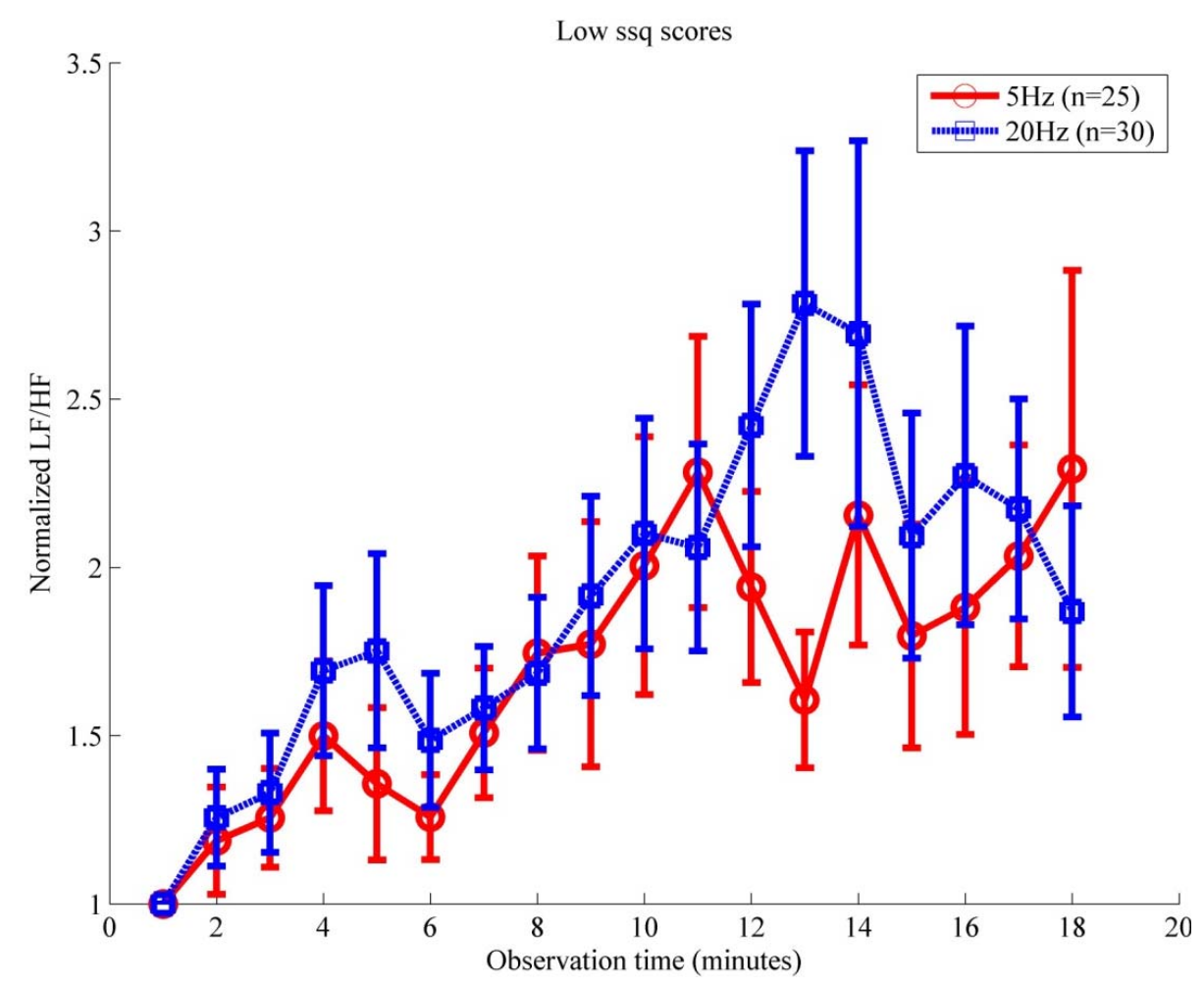

Figure 7. Change in the activity status of autonomic nervous system (LF/HF ratio) while observing the movie. Error bars indicate 1SE. Data of the subjects whose total scores of SSQ were less than average. 
activity status of autonomic nervous system significantly increased by observing the 3D movie given the vibration stimuli regardless of vibration frequency. The results suggested that there seem to be some threshold of comfort in the interaction between frequency and amplitude. This is considered important information to maintain visual health and comfort while watching stereoscopic movies.

\section{ACKNOWLEDGEMENTS}

The present study was conducted as a project of 2011 Japan-US collaboration for standardization on emerging technologies from the Ministry of Economy, Trade and Industry (METI), Japan. We would like to thank Hiromi Kamiiwa, Keiko Suzuki, and Seiko Fujisaki from the Health Research Institute at AIST for their assistance in analyzing the research data.

\section{REFERENCES}

[1] Chase, C., Tosha, C., Borsting, E. and Ridder III, W.H. (2009) Visual discomfort and objective measures of static accommodation. Optometry and Vision Science, 86, 883889. doi:10.1097/OPX.0b013e3181ae1b7c

[2] Hoffman, D.M., Girshick, A.R., Akeley, K. and Banks, M.S. (2008) Vergence-accommodation conflicts hinder visual performance and cause visual fatigue. Journal of Vision, 8, 31-30. doi:10.1167/8.3.33

[3] Kooi, F.L. and Toet, A. (2004) Visual comfort of binocular and 3D displays. Displays, 25, 99-108. doi:10.1016/j.displa.2004.07.004

[4] Nojiri, Y., Yamanoue, H., Hanazato, A., Emoto, M. and Okano, F. (2004) Visual comfort/discomfort and visual fatigue caused by stereoscopic HDTV viewing. Proceedings of SPIE IS \& T Electronic Imaging, 5291, 303-313.

[5] Seuntiens, P.J.H., Meesters, L.M.J. and Ijsselsteijn, W.A. (2005) Perceptual attributes of crosstalk in 3D images. Displays, 26, 177-183. doi:10.1016/j.displa.2005.06.005

[6] Speranza, F., Tam, W.J. and Renaud, R. (2006) Effect of disparity and motion on visual comfort of stereoscopic images. Proceedings of the SPIE, 6055, 94-103.

\section{doi:10.1117/12.640865}

[7] Wopking, M. (1995) Viewing comfort with stereoscopic pictures: An experimental study on the subjective effects of disparity magnitude and depth of focus. Journal of the Society for Information Display, 3, 101-103. doi:10.1889/1.1984948

[8] Yano, S., Ide, S., Mitsuhashi, T. and Thwaites, H. (2002) A study of visual fatigue and visual comfort for 3D HDTV/HDTV images. Displays, 23, 191-201. doi:10.1016/S0141-9382(02)00038-0

[9] Emoto, M., Niida, T. and Okano, F. (2005) Repeated vergence adaptation causes the decline of visual functions in watching stereoscopic television. Journal of Display Technology, 1, 328-340. doi:10.1109/JDT.2005.858938

[10] Emoto, M., Nojiri, Y. and Okano, F. (2004) Changes in fusional vergence limit and its hysteresis after viewing stereoscopic TV. Displays, 25, 67-76. doi:10.1016/j.displa.2004.07.001

[11] Ukai, K., Oyamada, H. and Ishikawa, S. (2000) Changes in accommodation and vergence following 2 hours of movie viewing through binocular head-mounted display. In: Franzen, O., Richter, H. and Stark, L., Eds., Accommodation and Vergence Mechanisms in the Visual System, Birkhauser Verlag, Switzerland, 313-325.

[12] Watanabe, H. and Umemura, U. (2008) Estimated degree of discomfort for instantaneous pulsation generated by motion base system: Psychological model and effect of predictive sign and subjects' attributions. Driving Simulation Conference, South Korea, 25 September 2008.

[13] Kennedy, R.S., Lane, N.E., Berbaum, K.S. and Lilienthal, M.G. (1993) Simulator sickness questionnaire: An enhanced method for quantifying simulator sickness. The International Journal of Aviation Psychology, 3, 203-220. doi:10.1207/s15327108ijap0303_3

[14] 3D Consortium (2011) 3DC Safety Guidelines. http://www.3dc.gr.jp/english/index.html

[15] Pölönen, M., Salmimaa, M., Takatalo, J. and Häkkinen, J. (2012) Subjective experiences of watching stereoscopic Avatar and U2 3D in a cinema. Journal of Electronic Imaging, 3, 5. doi:10.1007/3DRes.02(2012)5 